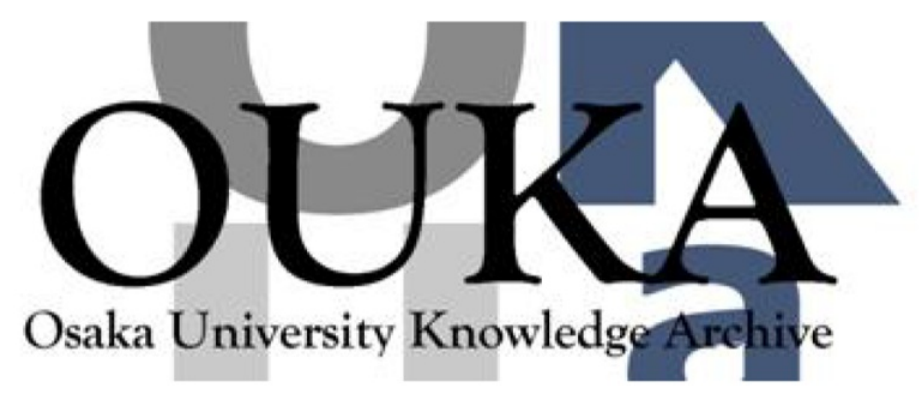

\begin{tabular}{|c|l|}
\hline Title & $\begin{array}{l}\text { Development of a Schwarzschild-type x-ray } \\
\text { microscope }\end{array}$ \\
\hline Author(s) & Kado, M.; Tanaka, K. A. ; Kodama, R. et al. \\
\hline Citation & Optics Letters. 16(2) p. 109-p. 111 \\
\hline Issue Date & $1991-01-15$ \\
\hline oaire:version VoR \\
\hline URL & https://hdl. handle. net/11094/2958 \\
\hline rights & \\
\hline Note & \\
\hline
\end{tabular}

Osaka University Knowledge Archive : OUKA

https://ir. Library. osaka-u. ac. jp/

Osaka University 


\title{
Development of a Schwarzschild-type x-ray microscope
}

\author{
M. Kado, K. A. Tanaka, R. Kodama, T. Yamanaka, and S. Nakai \\ Institute of Laser Engineering, Osaka University, 2-6 Yamada-oka, Suita, Osaka, 565, Japan
}

K. Yamashita, ${ }^{*}$ M. Ohtani, and S. Kitamoto

Department of Physics, Faculty of Science, Osaka University, 1-1 Machikaneyama-cho, Toyonaka, Osaka, 560, Japan

Received May 30, 1990; accepted November 5, 1990

\begin{abstract}
A Schwarzschild-type $\mathrm{x}$-ray microscope has been designed, constructed, and tested. $\mathrm{Ni} / \mathrm{C}$ multilayers were used as the $\mathrm{x}$-ray mirrors, with a thickness $(2 d)$ of $7 \mathrm{~nm}$ and 30 layer pairs. The microscope has attained a spatial resolution of $0.5 \mu \mathrm{m}$ at a magnification of 15 . By using bright laser-produced plasmas as an x-ray source [R. Kodama, K. Okada, N. Ikeda, M. Mineo, K. A. Tanaka, T. Mochizuki, and C. Yamanaka, J. Appl. Phys. 59, 3050 (1986)], images could be recorded during the 400 -psec laser pulse.
\end{abstract}

X-ray imaging optics have been essential diagnostics in inertial confinement fusion research. A micropellet ( $<1 \mathrm{~mm}$ diameter) is irradiated by a large multibeam laser system. This pellet is imploded to create a thermonuclear fusion condition, making use of the inertia of the imploding pellet wall. It is of critical importance for inertial confinement fusion to measure nonuniformities at the implosion core $(\sim 100 \mu \mathrm{m})$ or the laser spot in order to drive the pellet to a high density. Such measurements require a spatial resolution of less than $1 \mu \mathrm{m}$. Three types of $\mathrm{x}$-ray microscope have been typically known to offer fairly good resolution: Wolter, ${ }^{1,2}$ zone plate, ${ }^{3}$ and Schwarzschild ${ }^{4-8}$ types. We have chosen to build a Schwarzschild-type $x$-ray microscope with a magnification of 15. This $x$-ray microscope can be applied to fields other than laser fusion, such as lithography, ${ }^{6}$ biolo$\mathrm{gy}, 9,10$ and medicine. It can be a powerful tool when coupled with laser plasma $x$ rays (LAPLAX), since the high brightness and temporal resolution $(<1 \mathrm{nsec})$ of LAPLAX is added to the high spatial resolution.

Recently there have been several attempts to develop Schwarzschild $x$-ray microscopes. Some of these microscopes had a spatial resolution higher than 0.2 $\mu \mathrm{m}$ and diffraction-limited performance. ${ }^{7}$ However, most of the instruments use wavelengths exceeding 10 $\mathrm{nm}$, exhibit demagnified images, ${ }^{5-7}$ and use synchrotrons as x-ray sources. ${ }^{6,7}$ For the case of LAPLAX, multiple pulses were needed to obtain an image. ${ }^{5}$ In biology and medicine it is important to use a single pulse of LAPLAX, since high temporal resolution and minimum radiation damage are necessary. In addition, the shorter wavelength (at least less than $10 \mathrm{~nm}$ ) is desirable in $\mathrm{x}$-ray lithography.

In this Letter we present the development of a Schwarzschild-type $\mathrm{x}$-ray microscope. This microscope images with a magnification of 15 (Ref. 4) at an $\mathrm{x}$-ray wavelength of $7 \mathrm{~nm}$. With a bright LAPLAX, an exposure time of less than 1 nsec can be obtained.

Ray traces have been calculated for a working distance (the separation between the object point and the image point) of $1500 \mathrm{~mm}$ and a magnification of 15 . These values were determined by taking into account our implosion experimental conditions. Based on these calculations an optimum design was chosen, as shown in Table 1.

A spatial resolution was also calculated based on these parameters. In this calculation $x$-ray scattering due to the surface roughness of both mirrors was taken into account. The $\mathrm{x}$-ray scattering angle is given by ${ }^{11}$

$$
\Delta \theta_{s}= \pm 16 \pi^{2} \sigma^{2} / \lambda l_{0},
$$

where $\Delta \theta_{s}$ is the scattering angle, $\sigma$ is the rms height of the mirror surface roughness, $\lambda$ is the $x$-ray wavelength, and $l_{0}$ is the scale length of the mirror. Equation (1) indicates that the spatial resolution degrades as the x-ray wavelength becomes shorter and the rms height becomes larger. A ray-trace calculation showed that the spatial resolution had an almost linear dependence on the rms height. The best spatial resolutions were calculated for rms heights of 0.3 and $0.6 \mathrm{~nm}$. The value of $0.3 \mathrm{~nm}$ corresponds to the surface roughness of the quartz mirror substrate. The value of $0.6 \mathrm{~nm}$ corresponds to an estimated value of the interfacial roughness of the multicoated layers for the Schwarzschild-type $\mathrm{x}$-ray microscope as described below. For a height of $0.3 \mathrm{~nm}$ the best spatial resolution was $0.08 \mu \mathrm{m}$ within $\mathrm{a} \pm 100-\mu \mathrm{m}$ field of view. For a height of $0.6 \mathrm{~nm}$ the spatial resolution was $0.3 \mu \mathrm{m}$. The surface figures of the mirrors were measured by a Zygo Mark 4 interferometer, and the value was $\lambda / 10$ at

Table 1. Optimum Parameters of the SchwarzschildType X-Ray Microscope

\begin{tabular}{lcc}
$R_{1}$ (curvature of the concave mirror) & $344.962 \mathrm{~mm}$ \\
$D_{1}$ (diameter of the concave mirror) & $120 \mathrm{~mm}$ \\
$R_{2}$ (curvature of the convex mirror) & $116.445 \mathrm{~mm}$ \\
$D_{2}$ (diameter of the convex mirror) & $21.5 \mathrm{~mm}$ \\
$X$ (working distance) & 1500 & $\mathrm{~mm}$ \\
Magnification & 15 & \\
\hline
\end{tabular}




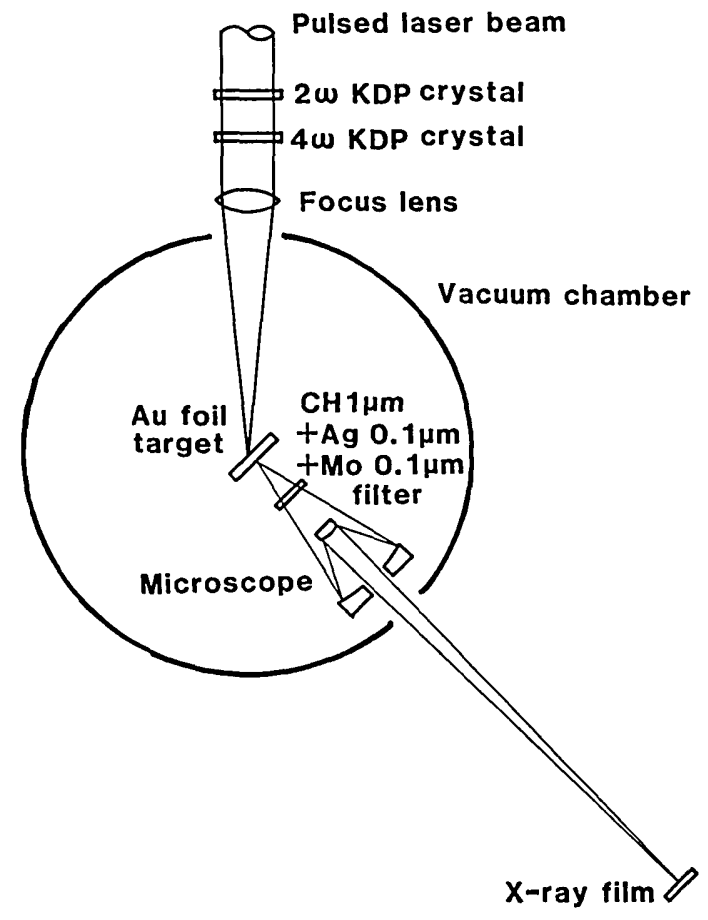

Fig. 1. Experimental setup. A 263-nm laser irradiates the Au target with 3-6 J of energy; the pulse duration is $400 \mathrm{psec}$, and the spot diameter is $200 \mu \mathrm{m}$. A 500- or 1000 -lines/in. mesh was attached to the rear side of the Au target. The Schwarzschild microscope observed the backlit image of the mesh, making use of the transmitting Au x rays.

a laser wavelength of $630 \mathrm{~nm}$. The role of the surface figure of the mirrors was also calculated. A deviation of $\lambda / 10$ from a perfect spherical surface degraded the best spatial resolution from 0.3 to $\sim 0.33 \mu \mathrm{m}$.

To produce the $\mathrm{x}$-ray mirrors, $\mathrm{Ni}$ and $\mathrm{C}$ layers were deposited onto quartz substrates by using an electronbeam evaporation method. ${ }^{12}$ With this method it is possible to form large uniform layers (as much as 150 $\mathrm{mm}$ in diameter). The surface roughness of the substrate was measured to be $0.3 \mathrm{~nm}(\mathrm{rms})$ by a WYKO optical heterodyne profilometer. The thicknesses of the $\mathrm{Ni}$ and $\mathrm{C}$ layers were 1.2 and $2.3 \mathrm{~nm}$, respectively, corresponding to an x-ray wavelength of $0.7 \mathrm{~nm}$. Many mirrors were test coated with a varying number of layer pairs (20 to 40 ). Test pieces were also coated at two substrate temperatures, room temperature and liquid $\mathrm{N}_{2}$ temperature.

The reflectivities were measured with $\mathrm{Cu} \mathrm{K} \alpha(0.154$ $\mathrm{nm}), \mathrm{Al} \mathrm{K} \alpha(0.834 \mathrm{~nm})$, and $\mathrm{C} \mathrm{K} \alpha(4.48 \mathrm{~nm})$ line x rays. The reflectivity at liquid $\mathrm{N}_{2}$ temperature could be two times higher than that at room temperature. The reflectivities depend on the number of layer pairs. A peak value was obtained with 30 layer pairs. For example, the reflectivity was $6 \%$ at the $\mathrm{Al} \mathrm{K} \alpha$ line $\mathrm{x}$ ray $(0.834 \mathrm{~nm})$. The observed reflectivities were always smaller than the calculated reflectivities. These differences may be attributed to a combination effect of interfacial roughness and interdiffusion between the $\mathrm{Ni}$ and $\mathrm{C}$ layers. The effective roughness was estimated to be $0.6 \mathrm{~nm}$. With this estimated value of interfacial roughness and interdiffusion of $0.6 \mathrm{~nm}$, the observed reflectivity was in good agreement with the calculated ones. Using the experimental and calculated incident angle dependences, we expect a reflectivity of $\sim 1-2 \%$ at normal incidence.

The spatial resolution was measured using LAPLAX. Figure 1 shows the experimental setup. The fourthharmonic light ( $263 \mathrm{~nm}$ ) of a Nd:glass laser was used to irradiate $0.2-\mu \mathrm{m}$-thick Au foil. The beam energy was 3-6 J, with a pulse width of 400 psec and a focal spot diamter of $200 \mu \mathrm{m}$. Under these laser conditions a high $x$-ray conversion efficiency $(\sim 80 \%)$ can be obtained, ${ }^{13}$ which provides a bright source of soft $x$ rays.

A $1-\mu \mathrm{m} \mathrm{CH}$ filter coated with $0.1 \mu \mathrm{m}$ of Mo and 0.1 $\mu \mathrm{m}$ of Ag was placed in front of the microscope in order to block plasma particles and strong scattered light. The soft-x-ray transmission of the filter was estimated to be $15 \%$ at $170 \mathrm{eV}$. Four filters were set on a rotating filter holder and could be selected without opening the vacuum chamber.

In this experiment a $0.2-\mu \mathrm{m}$-thick free-standing $\mathrm{Au}$ foil was used as an $\mathrm{x}$-ray target. The incident angle of the laser beam was $45^{\circ}$ with an $f / 10$ focus lens. $\mathrm{Cu}$ meshes of 500 and 1000 lines/in. (197 and 394 lines/ $\mathrm{cm}$ ) were attached on the rear side of the Au foil. The 500-lines/in. mesh has a opening of $30 \mu \mathrm{m}$ and a bar width of $20 \mu \mathrm{m}$. The 1000-lines/in. mesh has a opening of $15 \mu \mathrm{m}$ and a bar width of $10 \mu \mathrm{m}$. The $263-\mathrm{nm}$ laser irradiated the gold side, producing and transmitting an $x$-ray flux on the mesh. In this manner backlit mesh images were taken with the Schwarzschild-type

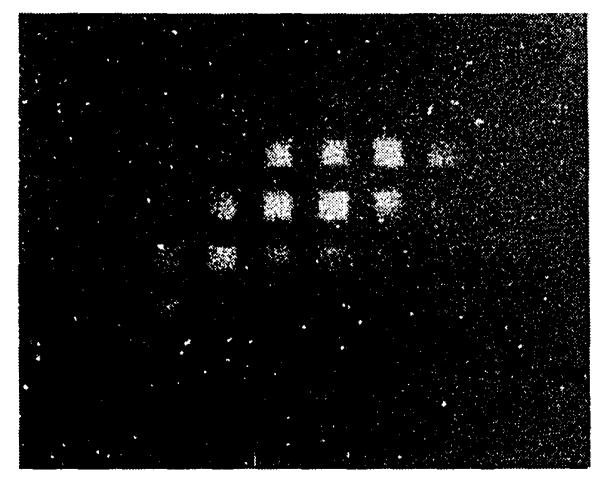

(a)

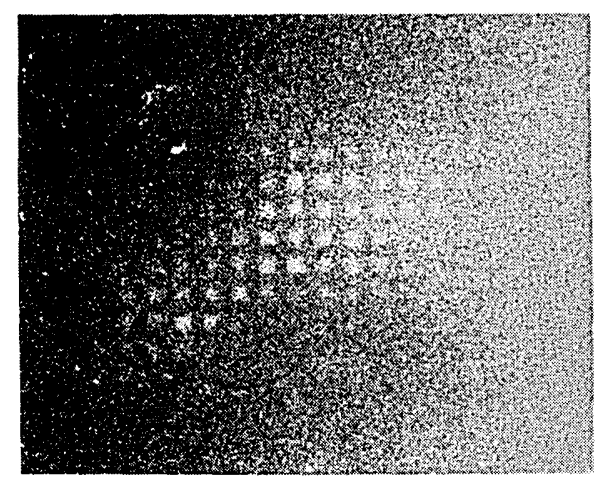

(b)

Fig. 2. Backlit mesh images with the microscope at $170 \mathrm{eV}$, with a mesh of (a) 500 lines/in. and (b) 1000 lines/in. 


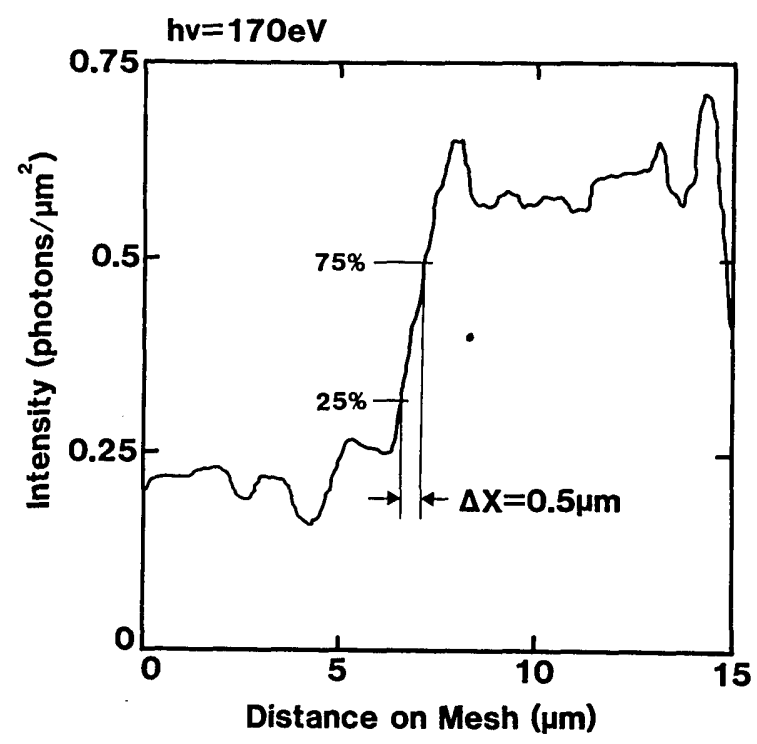

Fig. 3. Densitometer scan of the 1000-line/in. mesh image. With the $25-75 \%$ contrast, a spatial resolution of $0.5 \mu \mathrm{m}$ was achieved.

$\mathrm{x}$-ray microscope. The $\mathrm{x}$-ray images were recorded on Kodak 101-07 x-ray films.

Figures 2(a) and 2(b) show 170-eV x-ray backlit pictures of 500- and 1000-lines/in. meshes. The light and dark areas of the images correspond to the opening and the bars of the meshes. The 1000-lines/in. mesh image was scanned with a densitometer to produce the trace shown in Fig. 3. The spatial resolution, estimated at $25-75 \%$, was found to be $\sim 0.5 \mu \mathrm{m}$. This value might be an underestimation compared with a value found from a criterion of modulation transfer function $5 \%$.

This resolution was worse than the calculated value of $0.33 \mu \mathrm{m}$. The thickness of the mesh bars may be responsible for the degradation. The $\mathrm{x}$-ray flux is separated into three parts. One part is not obscured by the mesh bar. This area contributes to expose the $\mathrm{x}$-ray film. Another part is blocked perfectly by the mesh bar. This area corresponds to the dark area on the $x$-ray film. The third part is partly covered by the mesh bar. Thus this flux forms a gray area. It is this part that degrades the spatial resolution of the $x$-ray microscope. This size of the gray area was calculated to be $1.2 \mu \mathrm{m}$, with a half solid angle of $7^{\circ}$ of our microscope and a mesh bar size of $10 \mu \mathrm{m}$. In this experiment the mesh was attached to the $\mathrm{Au}$ foil. This calculated result shows that the spatial resolution degrades to $\sim 0.6 \mu \mathrm{m}$ by the obscuring effect of the mesh.

In summary, we have constructed a Schwarzschildtype $x$-ray microscope. Ray traces have been calculated, which took into account x-ray scattering due to the surface roughness and the role of the surface figure of both mirrors. The optimum design predicted a spatial resolution of $\sim 0.33 \mu \mathrm{m}$. Multilayer mirrors were fabricated for the microscope. The reflectivity of $6 \%$ at the $\mathrm{AI} \mathrm{K} \alpha$ line $\mathrm{x}$ ray could be obtained by cooling the substrate with liquid $\mathrm{N}_{2}$ during the electron-beam deposition. The microscope operates at a wavelength of $7 \mathrm{~nm}$ with a magnification of 15 . We have demonstrated the imaging capability of this microscope by recording images of $\mathrm{Cu}$ meshes attached to the $0.2-\mu \mathrm{m}-$ thick Au foil irradiated by $263-\mathrm{nm}$ pulsed laser beams. The backlit mesh images were recorded on $\mathrm{x}$-ray films. A spatial resolution of less than $0.5 \mu \mathrm{m}$ was determined from the data.

Part of this research has been supported by the Xray Image Optics Project of the Ministry of Education.

* Present address, Institute of Space and Astronautical Science, 1-1 Yoshinodai, 3 chome-Sagamihara, Kanagawa, 229, Japan.

\section{References}

1. S. Aoki and H. Yamaji, Jpn. J. Appl. Phys. 26, 1768 (1987).

2. S. Aoki, M. Shinozawa, K. Kanizaki, H. Hashimoto, M. Kakaji, Y. Setsuhara, H. Azechi, M. Yamanaka, T. Yamanaka, and C. Yamanaka, Jpn. J. Appl. Phys. 26, 952 (1987).

3. Y. Kagoshima, S. Aoki, M. Kakuchi, H. Maezawa, K. Hyodo, and M. Ando, in Forty-eighth Conference of Applied Physics (Japan Society of Applied Physics, Tokyo, 1988).

4. M. Kado, K. A. Tanaka, R. Kodama, A. Yamauchi, T. Mochizuki, K. Yamashita, T. Yamanaka, S. Nakai, and C. Yamanaka, Jpn. J. Opt. 17, 234 (1988).

5. J. A. Trail and R. L. Byer, Opt. Lett. 14, 539 (1989).

6. H. Kinoshita, T. Kaneko, H. Takei, N. Takeuchi, and N. Ishihara, in Forty-seventh Conference of Applied Physics, 28p-ZF-15 (Japan Society of Applied Physics, Tokyo, 1986).

7. D. W. Berreman, J. E. Bjorkholm, L. Eichner, R. R. Freeman, T. E. Jewell, W. M. Mansfield, A. A. MacDowell, M. L. O'Malley, E. L. Raab, W. T. Silfvast, L. H. Szeto, D. M. Tennant, W. K. Waskiewicz, D. L. White, D. L. Windt, O. R. Wood II, and J. H. Bruning, Opt. Lett. $15,529(1990)$.

8. D. L. Shealy, D. R. Gabardi, R. B. Hoover, A. B. C. Walker, Jr., J. F. Lindblom, and T. W. Barbee, Jr., J. XRay Sci. Technol. 1, 190 (1989).

9. M. Howells, J. Kirz, D. Sayre, and G. Schmahl, Soft-XRay Microscope (American Institute of Physics, New York, 1985).

10. S. Aoki, Oyo Buturi 56, 342 (1987).

11. H. Kunieda, S. Hayakawa, T. Hirano, T. Kii, F. Nagase, N. Sato, Y. Tawara, F. Makino, and K. Yamashita, Jpn. J. Appl. Phys. 25, 1292 (1986).

12. K. Yamashita, in Proceedings of International Meeting on Advanced Materials (Materials Research Society, Pittsburgh, Pa., 1989), Vol. 10, p. 221.

13. R. Kodama, K. Okada, N. Ikeda, M. Mineo, K. A. Tanaka, T. Mochizuki, and C. Yamanaka, J. Appl. Phys. 59, 3050 (1986). 